\title{
LuxO controls luxR expression in Vibrio harveyi: evidence for a common regulatory mechanism in Vibrio
}

Carol M. Miyamoto, ${ }^{1}$ Paul V. Dunlap, ${ }^{2}$ Edward G. Ruby ${ }^{3}$ and Edward A. Meighen ${ }^{1 *}$

${ }^{1}$ Department of Biochemistry, Room 813, McIntyre

Medical Sciences Building, McGill University, 3655

Promenade Sir William Osler, Montreal, Quebec, Canada, H3G 1 Y6.

${ }^{2}$ Department of Ecology and Evolutionary Biology, Kraus

Natural Science Building, University of Michigan, 830

North University Avenue, Ann Arbor, MI 48109-1048,

USA.

${ }^{3}$ Pacific Biomedical Research Center, University of

Hawaii, Manoa, 41 Ahui Street, Honolulu, HI 96813, USA.

\section{Summary}

Quorum-sensing control of luminescence in Vibrio harveyi, which involves an indirect autoinducermediated phosphorelay signal transduction system, contrasts with the prototypical quorum-sensing system of Vibrio fischeri, in which the autoinducer and the transcriptional activator LuxR directly activate lux operon expression. In $V$. harveyi, a regulator not homologous to $V$. fischeri LuxR and also designated LuxR (LuxR vh $_{\text {) }}$, binds specifically to the lux operon promoter region and activates the expression of luminescence. A direct connection has not been identified previously between $V$. harveyi $\operatorname{LuxR}_{\mathrm{vh}}$ and the autoinducer-mediated phosphorelay system. Here, we demonstrate by mobility shift assays and measurement of Iux $_{\text {vh }}$ mRNA levels with lux $O^{+}$and lux $O^{-}$cells that the central response regulator of the $V$. harveyi phosphorelay system (LuxO) represses the level of LuxR $\mathrm{vh}_{\mathrm{vh}}$. Expression of a lux $R_{v h}$-bearing plasmid strongly stimulated luminescence of a luxO mutant but had no effect on luminescence of wild-type lux $O^{+}$cells, indicating tight regulation of $\operatorname{lux}_{v h}$ by LuxO. Furthermore, luxO null mutants of $V$. fischeri MJ-1 and two autoinducer mutants, MJ-211 (IuxF) and MJ-215 (luxI ains ${ }^{-}$), emitted more light and exhibited more elevated levels of litR, a newly identified $V$. harveyi lux $R_{v h}$ homologue, than their luxO+ counterparts. These results suggest that activity of the autoinducer-

Accepted 30 December, 2002. *For correspondence. E-mail edward.meighen@mcgill.ca; Tel. $\quad(+1) \quad 514 \quad 398 \quad 7272 ; \quad$ Fax (+1) 5143987384 .

(C) 2003 Blackwell Publishing Ltd mediated phosphorelay system is coupled to LuxR $/$ LitR control of luminescence through LuxO in $V$. harveyi and $V$. fischeri. The presence of homologues of V. harveyi LuxR $\mathrm{vh}_{\mathrm{h}}$ LuxO and other phosphorelay system proteins in various Vibrio species and the control of LuxR $R_{\mathrm{vh}}$ and its homologues by LuxO identified here in $V$. harveyi and $V$. fischeri and recently in Vibrio cholerae suggest that the IuxO-IuxR $R_{v h}$ couple is a central feature of this quorum-sensing system in members of the genus Vibrio.

\section{Introduction}

Quorum sensing in prokaryotes has received much attention after the discovery that the luxl/R regulatory system controlling cell density-dependent expression of the Vibrio fischeri lux operon is found in many Gram-negative bacteria and acts as a global regulator for a number of functions ranging from virulence and natural competence to luminescence (Fuqua et al., 1994; Bassler, 1999; Swift et al., 1999; Joyce et al., 2000). The regulatory gene luxR is upstream and divergently transcribed from the lux operon, IUxICDABEG, and directs the synthesis of a transcriptional activator (LuxR). On interaction with the autoinducer N-(3-oxohexanoyl)-L-homoserine lactone (3oxo-C6-HSL), the product of the acyl-homoserine lactone (acyl-HSL) synthase (Luxl), LuxR activates the lux operon as well as its own gene (Engebrecht et al., 1983; Engebrecht and Silverman, 1984). A second autoinducer, $\mathrm{N}$-octanoyl-HSL (C8-HSL), the product of the acyl-HSL synthase (AinS), also interacts with LuxR to modulate luminescence via LuxR (Kuo et al., 1994; Gilson et al., 1995; Hanzelka et al., 1999).

A different quorum-sensing mechanism has been described in Vibrio harveyi, another luminescent species. The $V$. harveyi system lacks the IuxI/R circuitry but uses a phosphorelay signal transduction network (Bassler et al., 1993; 1994a) with two autoinducer signals: N-(3hydroxybutanoyl)-L-HSL (3-hydroxy-C4-HSL) (Al-1) (Cao and Meighen, 1989) and a nonacyl-compound (Al-2), recently shown to be a furanone (Schauder et al., 2001), specifically, a furanosyl borate ester (Chen et al., 2002). Syntheses of 3-hydroxy-C4-HSL and the furanosyl borate ester are dependent on LuxM and LuxS respectively. The presence of LuxS in many bacteria, both Gram-negative and Gram-positive, and its potential control of interspecies 
communication, have further stimulated interest in quorum sensing (Bassler, 1999).

Parallel sensory response pathways respond to the two autoinducer signals in $\mathrm{V}$. harveyi. The sensors for Al-1 and $\mathrm{Al}-2$ are LuxN and LuxQ (Freeman and Bassler, 1999a; Freeman et al., 2000), respectively, which can be phosphorylated with ATP at a specific histidine residue within the sensor recognition kinase domain followed by transphosphorylation to an aspartate residue in the downstream receiver domain. The phosphate is relayed from both sensors to the histidine-containing phosphate transfer domain in LuxU (Freeman and Bassler, 1999b), a detached phosphorelay protein, and then transferred to an aspartate residue in the response domain of the central response regulator LuxO (Freeman and Bassler, 1999a). The IuxU and luxO genes are closely linked with the $5^{\prime}$ terminal of luxU overlapping the 3 '-terminal of luxO. LuxO is a $\sigma^{54}$-binding protein with a DNA-binding domain that belongs to the NtrC family of $\sigma^{54}$-binding activators (Lilley and Bassler, 2000). The phosphorylated form (P-LuxO) at low cell densities is proposed to stimulate the $\sigma^{54}$ dependent expression of an unknown factor, $\mathrm{X}$, which in turn represses the expression of the $V$. harveyi luxCDA$B E G H$ operon. At high concentrations, Al-1 interacts with LuxN, and Al-2 associates with a periplasmic protein LuxP to interact with LuxQ (Bassler et al., 1994a; Schauder et al., 2001). These interactions cause the sensors to switch from kinase to phosphatase activity, leading to the dephosphorylation and inactivation of LuxO, thus permitting luminescence induction.

Conspicuously lacking in models of the quorum-sensing regulatory system of $V$. harveyi is the connection between $V$. harveyi LuxR $_{\mathrm{vh}}$ (not related to $V$. fischeri LuxR), a critical component for expression of the $V$. harveyi lux operon, and the autoinducer-mediated phosphorelay system. $\operatorname{LuxR}_{\mathrm{vh}}$ is a transcriptional activator that binds directly to the lux operon (Martin et al., 1989; Showalter et al., 1990; Swartzman et al., 1992; Swartzman and Meighen, 1993). The $V$. harveyi lux $R_{v h}$ null mutant MR1130 (Martin et al., 1989) does not produce luminescence even though its levels of autoinducers are normal. LuxR $\mathrm{Rh}_{\mathrm{vh}}$ has been suggested to be involved in the relay of the quorum-sensing signal (Miyamoto et al., 1996), but it is currently depicted in regulatory models for luminescence as an activator that functions independently of the quorum-sensing signals (Lilley and Bassler, 2000; Dunlap and Kita-Tsukamoto, 2001).

In this paper, we present evidence that LuxO represses lux $R_{v h}$ expression in $V$. harveyi through measurement of the levels of lux $R_{v h}$ mRNA by dot-blot hybridization, determination of LuxR $R_{v h}$ production by mobility shifts in regulatory mutants and comparison of the effects of expression with and without lux $R_{v h}$ in luxO null (luxO-) and wild-type cells. Recently, a parallel study of LuxO and HapR on toxicity in Vibrio cholerae has shown that LuxO represses hap $R$ expression at early stages of growth, which in turn blocks ToxR regulon expression (Zhu et al., 2002). HapR is a homologue of $V$. harveyi LuxR $R_{v h}$, both belonging to the TetR family of regulators (Jobling and Holmes, 1997). Because regulation of $L_{u x R}$ by LuxO in $V$. harveyi had not been demonstrated, Zhu et al. (2002) speculated that the regulation of HapR by LuxO is specifically related to the adaptation of $V$. cholerae to human host expression. We demonstrate, however, that LuxO functions by repression of not only LuxR $R_{v h}$ in $V$. harveyi but also a new $\operatorname{LuxR}_{\mathrm{vh}}$ homologue (LitR) in V. fischeri. LuxO control of LuxR $\mathrm{R}_{\mathrm{vh}}$ and its homologues may be a general regulatory mechanism in Vibrio.

\section{Results}

Dependence of lux $\mathrm{R}_{\mathrm{vh}} m R N A$ levels on LuxO

Previously, disruption of the $V$. harveyi luxO gene was shown to lead to a high constitutive level of luminescence independent of autoinducers. One explanation is that LuxO, as a $\sigma^{54}$ activator, regulates the synthesis of a $\sigma^{54}$ dependent repressor affecting the expression of the lux operon. Alternatively, LuxO might block LuxR $\mathrm{R}_{\mathrm{vh}}$ activation of the lux operon or inhibit synthesis or stability of $l u x R_{v h}$ mRNA (Lilley and Bassler, 2000). Accordingly, we investigated the level of lux $R_{v h}$ mRNA in $V$. harveyi wild-type and luxO strains, comparing luminescence and levels of lux $R_{v h}$ and IuxC mRNA. As was shown previously (Freeman and Bassler, 1999a), luminescence intensity was higher in luxO mutant than in wild-type cells early in growth (Fig. 1). The inhibition of light emission at low cell density in the luxO mutant reflects repression by components in the medium unrelated to quorum-sensing signals (Miyamoto et al., 2000; Chatterjee et al., 2002). Early in growth (Fig. 1, samples a-c), lux $0^{-}$cells contained more lux $_{v h}$ and I $u x C$ mRNA than the wild-type cells, whereas late in growth (Fig. 1, sample d, $A_{660}>1$ ), the mutant and wild-type cells contained the same levels of each of these transcripts. The levels of $\operatorname{lu} x C$ and $l u x R_{v h}$ mRNA cannot be compared directly as different labelled probes were used. Approximately seven times more luxC mRNA and three times more lux $R_{v h}$ mRNA were produced by the lux $\sigma^{-}$cells than by the wild-type cells at low cell densities $\left(A_{660} \geq 0.6\right)$ (Table 1). As a control, the mRNA for cyclic AMP receptor protein (CRP) (Chatterjee et al., 2002) was also assayed in wild-type and luxO cells at different stages of growth (Table 1). The levels of crp mRNA were equivalent in both wild-type and lux $0^{-}$cells at all stages of growth, representing constitutive production of message for CRP in contrast to that of luxC and lux $R_{v h}$ in wildtype cells. Moreover, the same level found for crp mRNA in the luxO null mutant as in the wild-type cells contrasts 

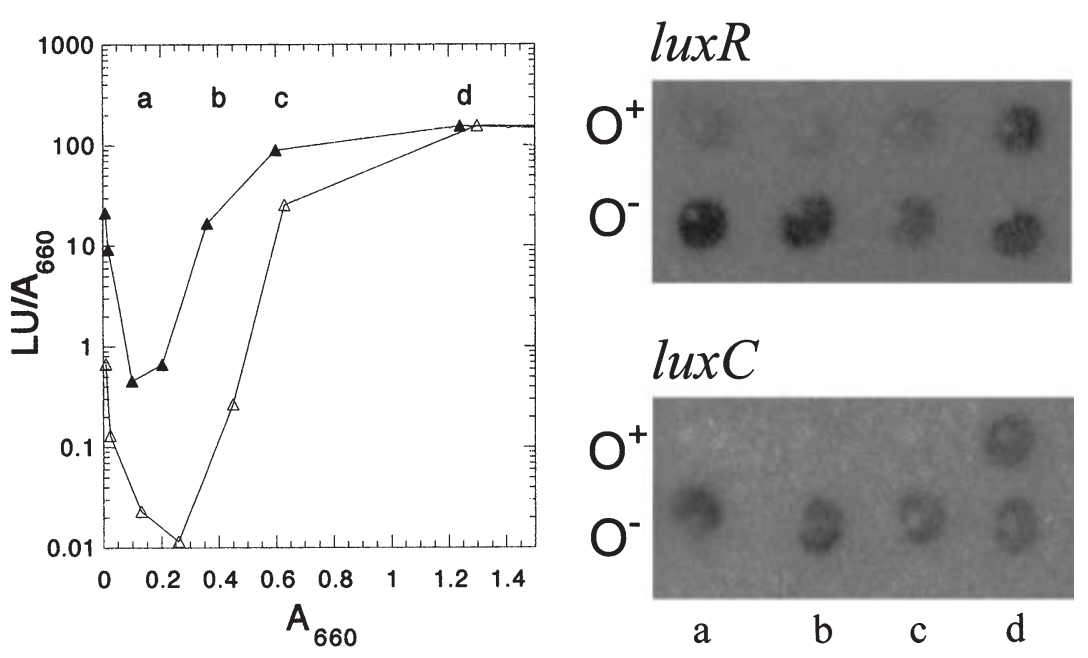

Fig. 1. Luminescence of $V$. harveyi wild type and the luxO null mutant and the levels of $\operatorname{lux}_{v h}$ and IuxC mRNA at various stages of cellular growth. Luminescence per unit $A_{660}$ $\left(\mathrm{LU} / \mathrm{A}_{660}\right)$ as a function of cellular density $\left(A_{660}\right)$ for $V$. harveyi wild type (open triangles) and the lux $\mathrm{O}^{-}$mutant (closed triangles) grown in complex medium is shown on the left. Periodically ( $a, b, c, d)$, cells were collected. After RNA extraction, RNA dot blots were prepared, and hybridization using ${ }^{32} \mathrm{P}$-labelled probes for $l u x R_{v h}$ and $l u x C$ was conducted. See Experimental procedures for further details. The autoradiogram of the resultant RNA dot blots of both wild-type and $\mathrm{LuxO}^{-}$ phenotypes is shown on the right. with the elevated levels of IuxC and IuxR $R_{v h}$ mRNA in the IuxO null mutant. Thus, disruption of luxO results in higher levels of lux mRNA including lux $R_{v h}$ mRNA at early stages of growth.

We next examined the $V$. harveyi autoinducer mutant D1 (Cao and Meighen, 1993), which lacks 3-hydroxy-C4HSL and furanosyl borate ester (Bassler et al., 1997; Chen et al., 2002) and produces a decreased level of lux $R_{v h}$ mRNA (Miyamoto et al., 1996). Disruption of luxO in $\mathrm{D} 1\left(\mathrm{D} 1 \mathrm{O}^{-}\right)$dramatically affected the level of luminescence, increasing it almost $3 \times 10^{5}$-fold (Fig. 2) to a level surpassing that of wild-type cells. Consistent with these

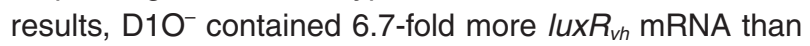
the D1 strain (Table 1). Furthermore, characteristic of luxO null mutants (Bassler et al., 1994b), exogenous addition of 3-hydroxy-C4-HSL had no effect on the lumines-

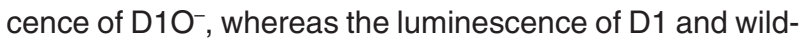
type was stimulated by this addition (Fig. 2).

\section{Measurement of LuxR $R_{v h}$ levels by mobility shifts}

To confirm that $l u x R_{v h}$ mRNA was being translated into active $\operatorname{LuxR}_{\mathrm{vh}}$, we used mobility shift assays of crude extracts from cells grown in LB medium to $A_{660}=0.6$ with labelled $l u x R_{v h}$ promoter DNA to assess $L_{u x R_{v h}}$ binding. Two bands migrating more slowly than the $l u x R_{v h}$ DNA can readily be observed (Fig. $3 A$ ), reflecting the two $L u x R_{v h}$ binding sites in the lux $R_{v h}$ promoter (Chatterjee et al., 1996). These mobility shifts resulted from LuxR $R_{v h}$ binding, as Fig. 3B (lane 2) shows that a crude extract of MR1130, the lux $R_{v h}$ null mutant of $V$. harveyi BB7, gave rise to a minor bandshift, which mirrored that seen using the control extract of Escherichia coli bearing the plasmid pT7 (Fig. 3B, lane 5). Using a positive control of an E. coli extract bearing $l u x R_{v h}$ in $\mathrm{pT} 7$ (pT7/lux $\mathrm{R}_{\mathrm{vh}}$ ), two major bandshifts attributed to $\operatorname{LuxR}_{\mathrm{vh}}$ binding to its own promoter (Chatterjee et al., 1996) migrate to the same positions in the gel as the reference sample (Fig. 3B, lane 8), which is reproduced in Fig. $3 \mathrm{~A}$ (lane 2). The stronger mobility shifts with extracts of the luxO mutants than with extracts of wild-type $V$. harveyi cells indicated that a higher level of LuxR $\mathrm{R}_{\mathrm{vh}}$ was present in the luxO- mutant, consistent with the relative levels of luxR $R_{v h}$ mRNA (Fig. 1). Using D1 extracts, the mobility shifts were even more dramatic; migration of very little DNA was retarded with $1 \mu \mathrm{g}$ of the $\mathrm{D} 1\left(\mathrm{ux} \mathrm{O}^{+}\right)$extract, whereas most of the DNA migrated

Table 1. Quantification of mRNAs in various luxO and luxO cells of $V$. harveyi and V. fischeri at different stages of growth ${ }^{2}$.

\begin{tabular}{llccc}
\hline Cells & mRNA & \multicolumn{1}{c}{$\mathrm{A}_{660^{b}}$} & $\mathrm{O}^{+}$(c.p.m.) & $\mathrm{O}^{-}$(c.p.m.) \\
\hline V. harveyi & luxC & $0.02-0.6$ & $79 \pm 19$ & $570 \pm 83$ \\
V. harveyi & luxC & $1.0-1.8$ & $567 \pm 104$ & $584 \pm 92$ \\
V. harveyi & luxR & $0.02-0.6$ & $169 \pm 41$ & $507 \pm 124$ \\
V. harveyi & luxR & $1.0-1.8$ & $557 \pm 81$ & $656 \pm 93$ \\
V. harveyi & crp & $0.08-0.5$ & $495 \pm 47$ & $641 \pm 160$ \\
V. harveyi & crp & $0.6-2.2$ & $395 \pm 134$ & $431 \pm 20$ \\
V. harveyi D1 & luxR & $0.6-2.0$ & $112 \pm 54$ & $748 \pm 212$ \\
V. fischeri & litR & $0.3-1.2$ & $387 \pm 109$ & 1.0 \\
V. fischeri & litR & $1.3-2.5$ & $2693 \pm 616$ & $2172 \pm 479$ \\
\hline
\end{tabular}

a. After growth in complex medium as in Fig. 1, cells were obtained and RNA prepared. RNA dot-blot hybridizations were quantified using the Fuji Bioimager, and c.p.m. after background subtraction is given. Three to six samples were used in each case.

b. A range of $A_{660}$ was used as the samples had similar amounts of radioactive probe bound. 


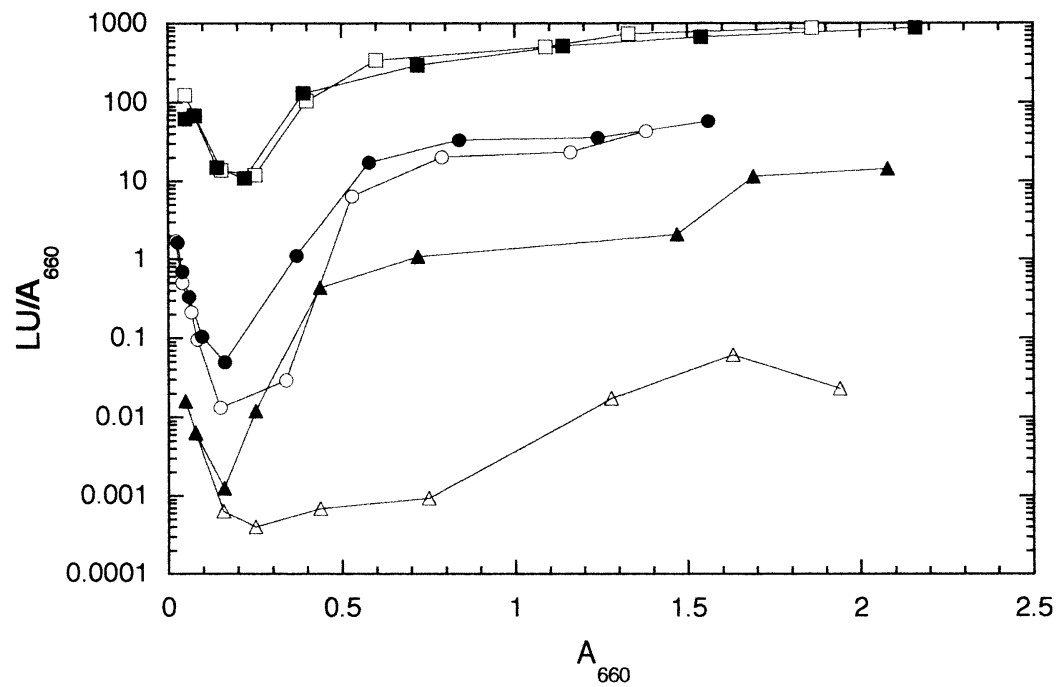

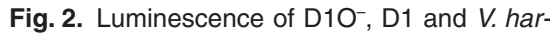
veyi wild type during growth with and without added 3-hydroxy-C4-HSL. Wild-type V. harveyi, the dark mutant D1 of $V$. harveyi and the luxO

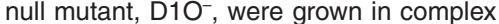
medium to obtain the plot of luminescence (LU/ $\left.A_{660}\right)$ with cell density $\left(A_{660}\right)$ in the presence and absence of 3-hydroxy-C4-HSL at $10 \mu \mathrm{g} \mathrm{ml}^{-1}$. Wild type without 3-hydroxy-C4-HSL (open circles), with 3-hydroxy-C4-HSL (closed circles); D1 without 3-hydroxy-C4-HSL (open triangles), with 3-hydroxy-C4-HSL (closed triangles); D10- without 3-hydroxy-C4-HSL (open squares), with 3-hydroxy-C4-HSL (closed squares).

more slowly with $1 \mu \mathrm{g}$ of the $\mathrm{D}^{-} \mathrm{O}^{-}$extract. These results demonstrate a direct correlation between higher levels of $\operatorname{luxR}_{v h}$ mRNA and higher levels of LuxR $\mathrm{R}_{\mathrm{vh}}$ (see Table 1). Finally, when $V$. harveyi MR1130, the lux $R_{v h}$ null phenotype, was made luxO by gene replacement (Experimental procedures) and examined for light production, the dark phenotype of the mutant remained unchanged (data not shown). In Fig. 3B, an extract of the luxO/ux $R^{-}$mutant $\left(\mathrm{MRO}^{-}\right)$subjected to mobility shift analysis with $l u x R_{v h}$ promoter DNA gave rise to the same minor band as seen in both extracts of MR1130 and E. coli(pT7) cells; with five times more $\mathrm{MRO}^{-}$extract, the intensity of this minor band remained unchanged. These negative findings for luminescence and $\mathrm{LuxR}_{\mathrm{vh}}$ in $\mathrm{MRO}^{-}$suggest that no other component that may be under LuxO control appears to enable luminescence when LuxR $_{v h}$ is absent.

\section{Expression of lux $\mathrm{R}_{\mathrm{vh}}$ in trans in luxO null mutants}

Previously, we demonstrated that the presence of $l u x R_{v h}$ in trans in D1 restored luminescence to wild-type levels bypassing the requirement for the addition of autoinducers (Miyamoto et al., 1996). To examine the effect of multiple copies of lux $R_{v h}$ in the absence of LuxO, we transferred the luxR $R_{v h}$-bearing plasmid (pMGM150) into both wild-type and luxO strains. Cells were grown in LB, which is necessary to maintain good growth with a plasmid present (Fig. 4). The typical repression of luminescence early in growth was observed, which was not alleviated by the presence of pMGM150 in wild-type cells, but the weaker repression of luminescence observed in luxO cells was almost completely overcome in the presence of pMGM150. Indeed, at earlier stages of growth, luminescence was 10 times higher in the luxO (+lux $\left.R_{v h}\right)$ cells than in the lux $O^{-}$cells and 100 times higher than in wild-type $\left( \pm / u x R_{v h}\right)$ cells. To ensure that the $\operatorname{lux}_{v h}$ plasmid was being expressed in both cell types, extracts (from cells grown to $A_{660}=0.6$ in LB medium) were assayed for their ability to cause the LuxR $R_{v h}$ mobility shifts using labelled lux $R_{v h}$ promoter DNA (Fig. 3B). When the lux $R_{v h}$ plasmid was present in wild-type and lux $0^{-}$cells, both extracts

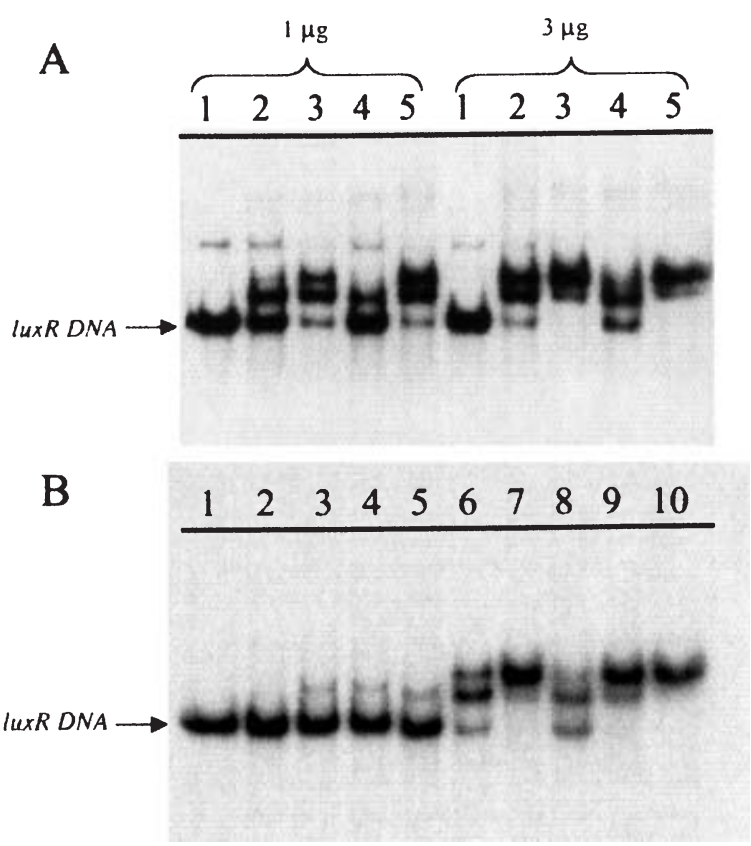

Fig. 3. Levels of LuxR $R_{v h}$ as determined by mobility shifts. Autoradiograms after $5 \%(\mathrm{w} / \mathrm{v})$ PAGE of $V$. harveyi $5^{\prime}{ }^{32} \mathrm{P}$-labelled lux $R_{v h}$ promoter DNA (400 bp, as described in Experimental procedures) mixed with (A) 1 or $3 \mu \mathrm{g}$ of protein lysate: 1 , no addition; $2, V$. harveyi wild type; $3, \mathrm{VhO}^{-}$, the luxO null mutant; 4, D1; 5, D10-; (B) $1 \mu \mathrm{g}$ of protein lysate for all except lane $4(5 \mu \mathrm{g})$, lane $6(0.01 \mu \mathrm{g})$ and lane 7 $(0.05 \mu \mathrm{g}): 1$, no addition; 2, MR1130; 3, $\mathrm{MRO}^{-} ; 4, \mathrm{MRO}^{-} ; 5$, E. coli BL21(pT7); 6, E. coli BL21(pT7/luxR $\left.R_{v h}\right) ; 7$, E. coli BL21(pT7//uxR $\left.R_{v h}\right) ;$ 8, V. harveyi wild type; 9, V. harveyi lux $O^{+}$(+lux $R_{v h}$ [pMGM150]); 10 , $V$. harveyi luxO- (+lux $R_{v h}$ [pMGM150]). All lysates were prepared from cells grown in LB medium to $A_{660}=0.6$. 


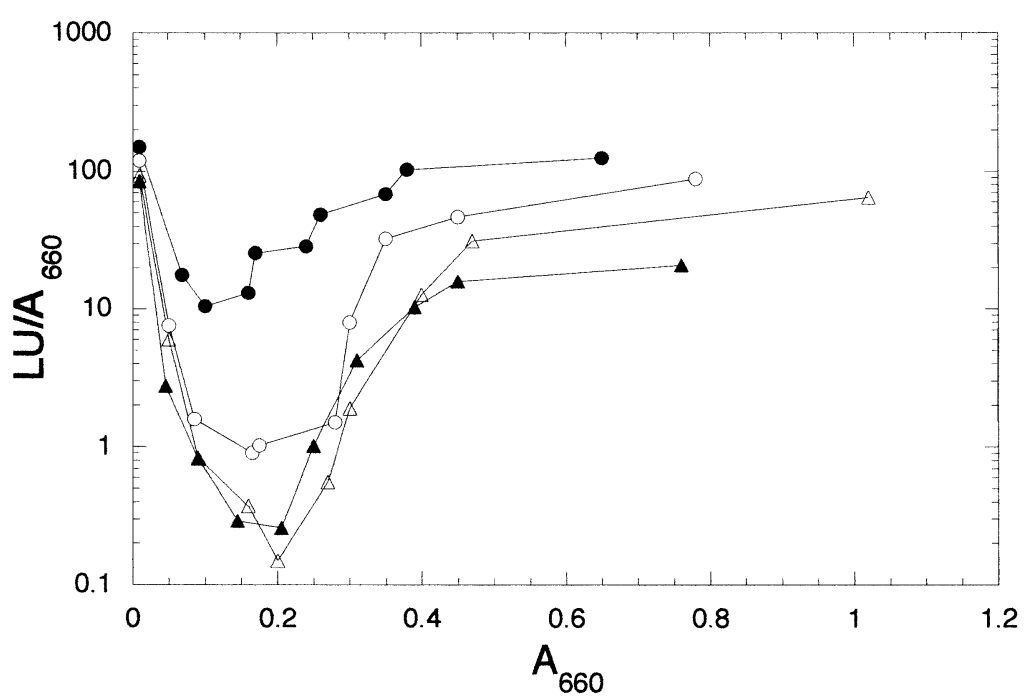

Fig. 4. Luminescence with cellular growth of $V$. harveyi wild type and the luxO null mutant with and without in trans addition of $V$. harveyi lux $R_{v h}$. The mobilizable plasmids $\mathrm{pKT} / / \mathrm{ux} \boldsymbol{R}_{v h}$ (pMGM150) or pKT230 were introduced to wildtype and luxO cells, and the luminescence $\left(\mathrm{LU} / \mathrm{A}_{660}\right)$ was measured at various times during growth $\left(\mathrm{A}_{660}\right)$ in LB medium: wild type + pKT230 (open triangle), wild type + pMGM150 (closed triangle); luxO + pKT230 (open circle); luxO + pMGM150 (closed circle). caused a very strong mobility shift of the lux $R_{v h}$ promoter

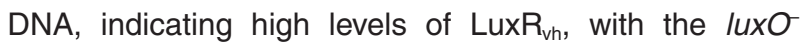
extract showing the strongest binding (Fig. 3B). Although the levels of functional LuxR $_{v h}$ in vivo are difficult to define, particularly with the presence of multicopy plasmid capable of binding $\operatorname{LuxR}_{\mathrm{vh}}$, the ability of luxR $R_{v h}$ in trans to

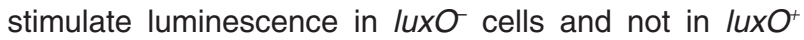
cells provides strong support that $l u x O$ regulates $I u x R_{v h}$.

\section{Polyhydroxybutyrate production in luxO null mutants}

In $V$. harveyi, it has been demonstrated that $L_{u x} R_{v h}$ controls the production of polyhydroxybutyrate (PHB) at late stages of growth (Miyamoto et al., 1998). To test whether a phenotype controlled by $\operatorname{LuxR}_{\mathrm{vh}}$ other than luminescence is also affected when LuxO is absent, we analysed D1 and MR1130 cells and their respective luxO null cells for PHB (Experimental procedures). Both D1 and MR1130 have been determined to lack PHB because of the insufficiency or absence, respectively, of $L u x R_{v h}$. As expected, the level of crotonic acid produced by oxidation of $\mathrm{PHB}$ was not detectable in D1 or MR1130 cells (Fig. 5). How-

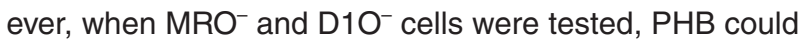
still not be detected in the luxR-negative $\mathrm{MRO}^{-}$cells, whereas a large amount of PHB was produced in the

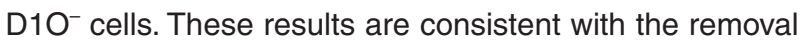
of the repression of $l u x R_{v h}$ by disrupting luxO and demonstrate that the luxO-lux $R_{v h}$ regulatory couple controls multiple phenotypes.

$L u x O$ regulation of $L i t R$, a V. harveyi $L u x R_{v h}$ homologue in V. fischeri

LuxO has been identified in $V$. fischeri as well, where it

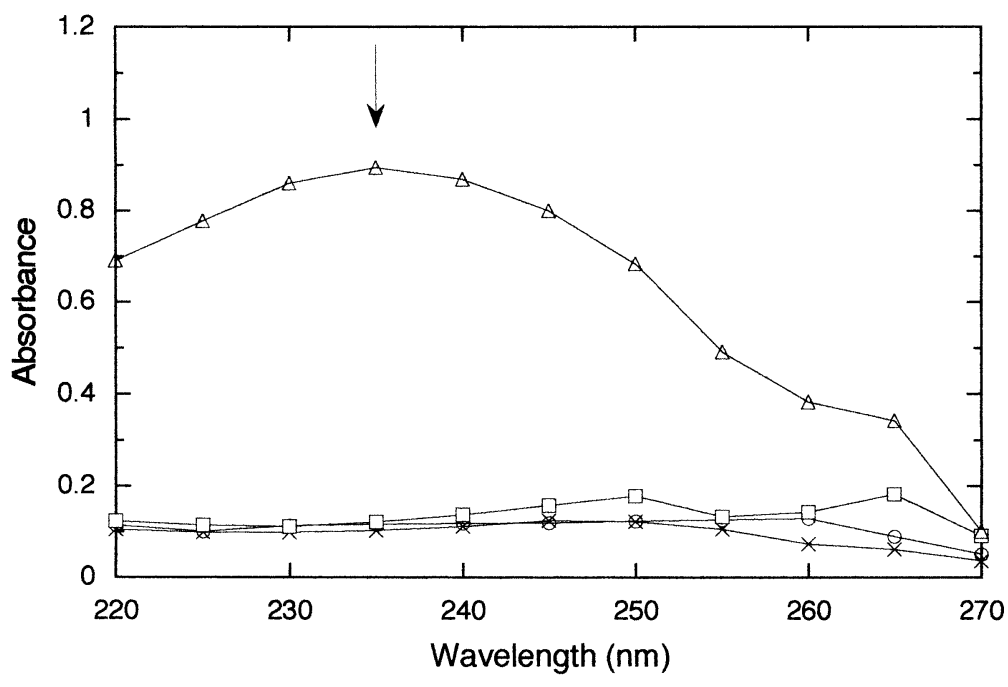

(C) 2003 Blackwell Publishing Ltd, Molecular Microbiology, 48, 537-548
Fig. 5. PHB production in MR1130, D1 and their luxO counterparts. The oxidation of $\mathrm{PHB}$ into crotonic acid was measured as absorbance vs. wavelength. The maximum wavelength for crotonic acid $(235 \mathrm{~nm})$ is indicated. The scans of absorbance from 220 to $270 \mathrm{~nm}$ were measured in a Beckman DU 640 spectrophotometer: MR1130 (X); $\mathrm{MRO}^{-}$(circle); D1 (square); $\mathrm{D}^{-} \mathrm{O}^{-}$(triangle). 
also controls luminescence (Miyamoto et al., 2000). The quorum-sensing systems controlling luminescence in $V$. harveyi and $V$. fischeri, however, appear to be distinctly different, leading to questions on the mechanism by which LuxO operates in $V$. fischeri. The recent discovery of a $V$. harveyi lux $R_{v h}$ homologue, litR, in $V$. fischeri ES114 (Fidopiastis et al., 2002) allowed us to address this issue. Specifically, to determine whether LuxO operates via LitR in $V$. fischeri, we quantified litR mRNA in $V$. fischeri MJ-1 (wild type) and its luxO mutant. Levels of litR mRNA were threefold higher in the luxO null mutant compared with $\mathrm{MJ}$ 1 in the early stages of growth $\left(\mathrm{A}_{660}<1.3\right)$, whereas lith mRNA levels were similar in these strains at higher cell densities (Table 1). The similarity of these results to those obtained with $V$. harveyi (Table 1) suggests that litR in $V$. fischeri and lux $R_{v h}$ in $V$. harveyi carry out the same function. To assess this possibility further, we mobilized the $V$. harveyi lux $R_{v h}$ gene into $V$. fischeri. The presence of $l u x R_{v h}$ strongly stimulated luminescence in the $V$. fischeri luxO null mutant but had no effect in MJ-1 (Fig. 6). These results again are very similar to those observed for the effect of lux $R_{v h}$ in $V$. harveyi (Fig. 4).

We next examined the effect of luxO in $\mathrm{V}$. fischeri in the presence and absence of quorum-sensing signals to assess the possibility that LuxO may function in this bacterium in a manner analogous to its autoinducer-mediated activity in $V$. harveyi. The $V$. fischeri acyl-HSL synthase mutants, MJ-211 (IuxI), which does not produce 3-oxo-C6HSL but produces C8-HSL, and MJ-215 (Iuxl, ainS), which produces neither 3-oxo-C6-HSL nor C8-HSL (Kuo et al., $1994 ; 1996)$, were made luxO by gene replacement (Experimental procedures). MJ-211 produces $\approx 100$-fold more light than MJ-215 because of the ability of C8-HSL, a competitive inhibitor of 3-oxo-C6-HSL, to activate LuxR weakly (Kuo et al., 1996). We found that the luxO mutants of MJ-211 and MJ-215 in each case produced 10-50 times more light than their parent strains (Fig. 7). These results demonstrate that LuxO represses luminescence in $V$. fischeri in the absence of either 3-oxo-C6-HSL or both 3-0xo-C6-HSL and C8-HSL.

As Ains is a homologue of LuxM that produces 3hydroxy-C4-HSL to overcome repression of luminescence by LuxO in V. harveyi, we added the AinS inducer, C8HSL, to deduce whether it could stimulate light not only by interaction with LuxR but by overcoming the repression of litR expression caused by LuxO. As expected, C8-HSL stimulated luminescence in all cell lines (Fig. 7). Moreover, in both lux 5 and luxtains cell lines, the degree of luminescence stimulation by $\mathrm{C} 8-\mathrm{HSL}$ is larger for the luxO $\mathrm{O}^{+}$cells than for the luxO cells, with the same level of luminescence being reached for the luxO+ and luxOstrains of the lux 5 ains cells. This result may suggest a link between LuxO repression and C8-HSL activation of luminescence, although interpretation of these data is complicated by the stimulation of luminescence by the interaction of C8-HSL with LuxR.

To examine this possible link more directly, we quantified litR mRNA in MJ-1 (wild-type), MJ-211 and MJ-215 and their luxO null mutants grown in the presence and absence of C8-HSL. In the absence of added autoinducer, a higher stimulation of litR mRNA $\left(\mathrm{O}^{-} / \mathrm{O}^{+}\right)$was observed for $\mathrm{MJ}-1$ in complex medium (threefold, Table 1) than in the ASH medium (twofold, Table 2). A similar fold increase was observed with $\mathrm{MJ}-215$ and its luxO mutant in $\mathrm{ASH}$ medium (1.8-fold), whereas the increase in the amount of litR mRNA in luxO- cells in MJ-211 was relatively low and possibly not significant. Unfortunately, MJ-211 and MJ215 only exhibit high luminescence in ASH medium and not in complex medium, in which wild-type cells gave the highest change in litR mRNA levels in response to the I $u x O$ mutation. For MJ-1, addition of C8-HSL, or elimination of LuxO, resulted in a twofold increase in litR mRNA;

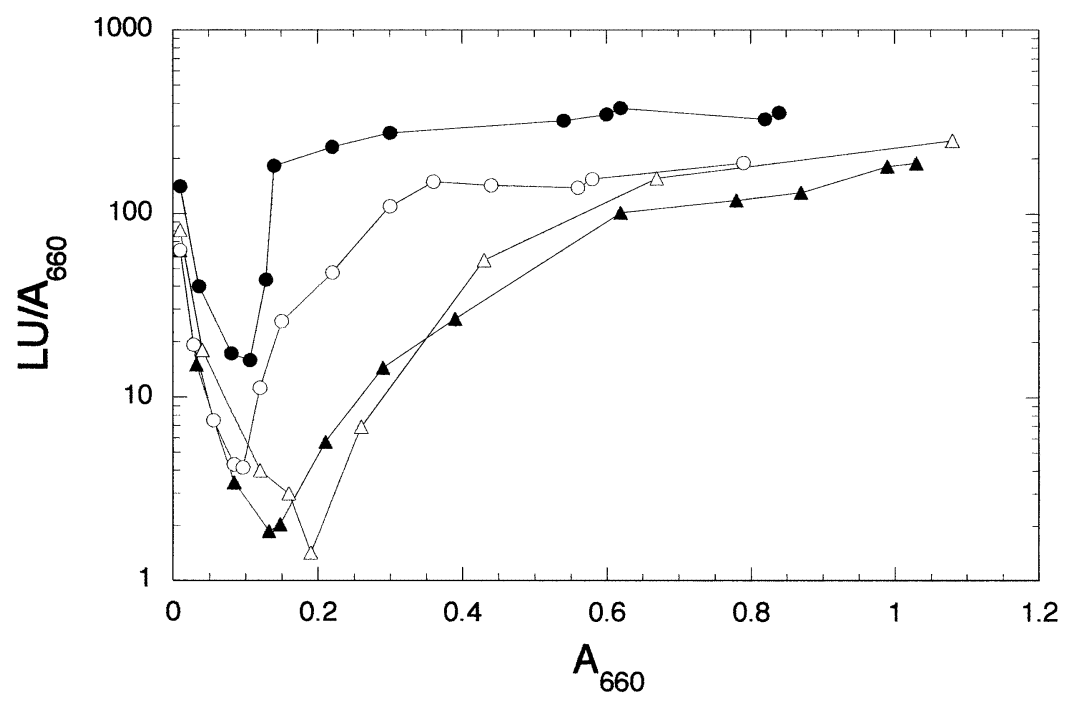

Fig. 6. Effect of in trans addition of $V$. harveyi lux $R_{v h}$ to $V$. fischeri wild-type and luxO cells on cell density-dependent luminescence. Growth and luminescence were done as described in the legend to Fig. 4. V. fischeri wild type + pKT230 (open triangle), wild type + pMGM150 (closed triangle); luxO + pKT230 (open circle), luxO- pMGM150 (closed circle). 

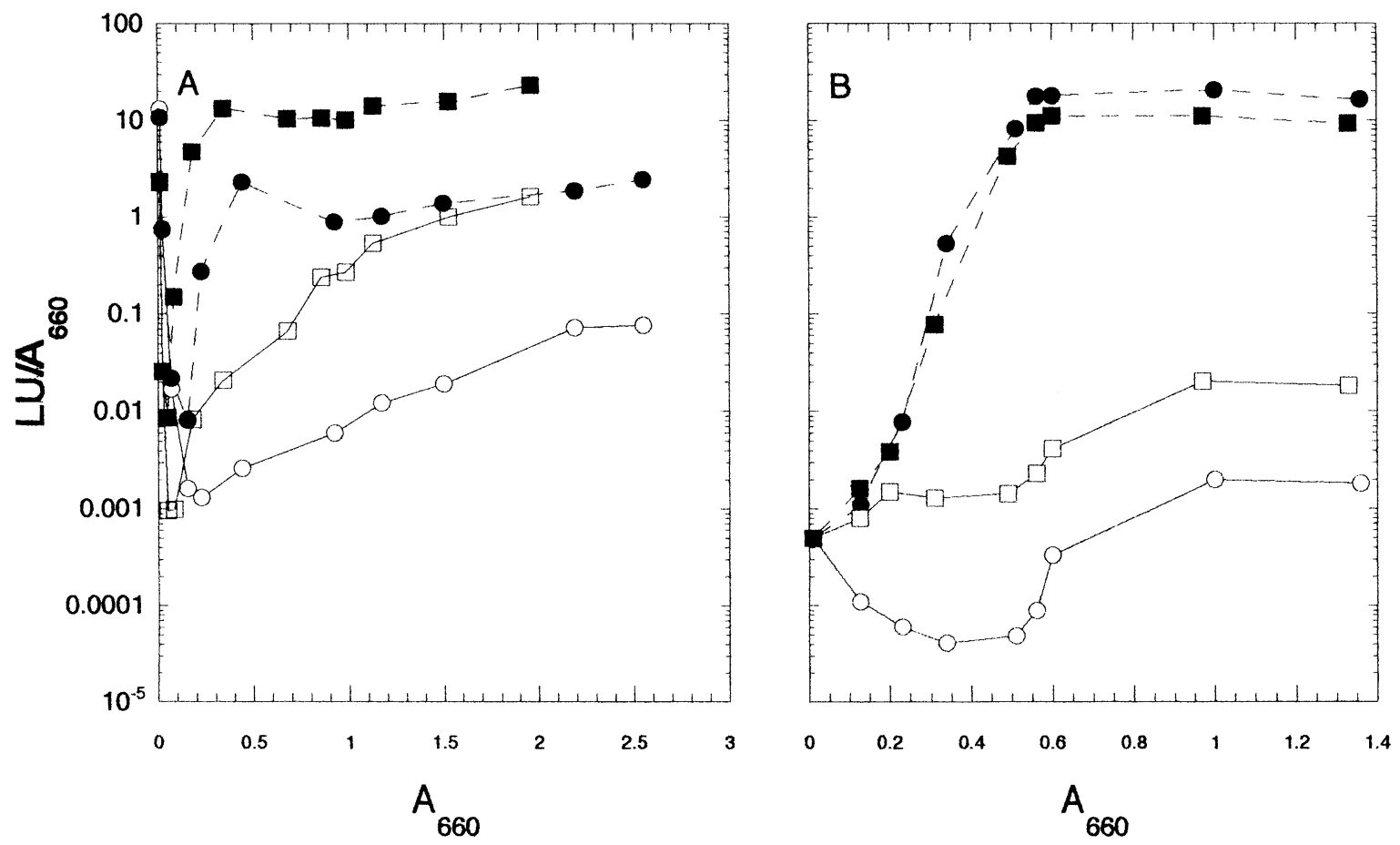

Fig. 7. Luminescence profiles during growth in ASH medium of $V$. fischeri autoinducer mutants (lux $O^{+}$and luxO $)$in the presence and absence of C8-HSL.

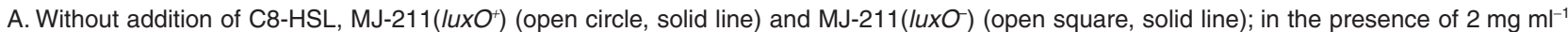

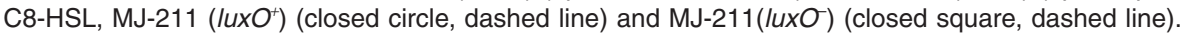

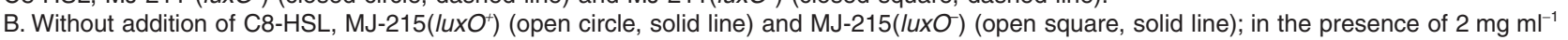
C8-HSL, MJ-215 (luxO+) (closed circle, dashed line) and MJ-215 (luxO) (closed square, dashed line).

however, the addition of C8-HSL had no effect on the luxO mutant of MJ-1 (Table 2). The lack of an effect of C8-HSL on the levels of litR mRNA in the three luxO null mutants of $V$. fischeri indicates that LuxO may be necessary for the response to this quorum-sensing signal. These results are very similar to those obtained for the effects of LuxO and 3-hydroxy-C4-HSL on luminescence (Bassler et al., 1994b) and luxR $R_{v h}$ mRNA in V. harveyi (Miyamoto et al., 1996).

Table 2. Levels of litR mRNA in luxO+ and luxO genotypes of various $V$. fischeri cells at mid-log phase of growth in the presence and absence of C8-HSL.

\begin{tabular}{llll}
\hline Cells $^{\mathrm{a}}$ & $\mathrm{O}^{+}$(c.p.m.) & $\mathrm{O}^{-/}$ & $\mathrm{O}^{+\mathrm{b}}$ \\
\hline $\mathrm{MJ}-1^{\mathrm{c}}$ & $1656 \pm 355$ & $3261 \pm 397$ & 2.0 \\
$\mathrm{MJ}-1+\mathrm{C} 8-\mathrm{HSL}$ & $3764 \pm 1439$ & $3468 \pm 681$ & 0.9 \\
$\mathrm{MJ}-211$ & $1951 \pm 308$ & $2524 \pm 389$ & 1.3 \\
$\mathrm{MJ}-211+\mathrm{C} 8-\mathrm{HSL}$ & $2184 \pm 504$ & $1882 \pm 411$ & 0.9 \\
$\mathrm{MJ}-215$ & $1064 \pm 138$ & $1928 \pm 279$ & 1.8 \\
$\mathrm{MJ}-215+\mathrm{C} 8-\mathrm{HSL}$ & $1534 \pm 177$ & $1628 \pm 176$ & 1.1 \\
\hline
\end{tabular}

a. Samples of cells grown in ASH medium from $A_{660}=0.3-1.0$ were used; when required, C8-HSL was added at $2 \mu \mathrm{g} \mathrm{ml}^{-1}$.

b. Ratio of litR mRNA in luxO and luxO cells.

c. MJ-1 was used instead of the parent $\mathrm{MJ}-100$ as both have identical phenotypes.

(C) 2003 Blackwell Publishing Ltd, Molecular Microbiology, 48, 537-548

\section{Discussion}

The systems regulating luminescence in bacteria are of great interest as they represent the prototypes for acylHSL-based quorum-sensing systems found in many pathogenic bacteria controlling secondary metabolism. The quorum-sensing system in $V$. harveyi involves signal transduction via phosphorelay proteins that transmit the signal from two different autoinducers, 3-hydroxy-C4-HSL and a furanosyl borate ester. Although LuxR $\mathrm{R}_{\mathrm{vh}}$ is the primary activator of the lux operon in $V$. harveyi binding to two sites in the luxCDABEGH promoter region (Swartzman et al., 1992; Miyamoto et al., 1994), its connection, if any, to the quorum-sensing system has been unclear. Previously, the strongest data supporting the involvement of $L u x R_{v h}$ in quorum sensing were the twofold stimulation of lux $R_{v h}$ expression by 3-hydroxy-C4-HSL (Miyamoto et al., 1996); however, these results did not establish its linkage to the phosphorelay components involved in the quorum-sensing regulatory system even though it has been speculated that both LuxN and LuxO may be able to transmit signals to lux $R_{v h}$ (Bassler et al., 1993; 1994a).

In the present paper, we have shown that lux $R_{v h}$ expression is repressed by LuxO in $V$. harveyi and provide evi- 
dence that a similar regulatory control also occurs for lit $R$ in $V$. fischeri. Enhanced lux $R_{v h}$ mRNA production (threefold) and LuxR $\mathrm{R}_{\mathrm{vh}}$ levels were clearly evident in the luxOmutant of $V$. harveyi at low cell density compared with those in wild-type cells, and paralleled the level of luxC mRNA in luxO- cells, which was about sevenfold higher. Luminescence was restored in an autoinducer-negative mutant (D1) with a luxO knock-out (D1/uxO-) and, at midlog phase, the level of lux $R_{v h}$ mRNA in D1/ux $0^{-}$was seven times higher than that of D1, the Iux $R_{v h}$ mRNA of which is lower than that of wild type. The larger increase in lux $R_{v h}$

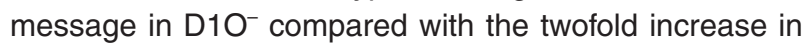
luxR $_{v h}$ mRNA observed on adding 3-hydroxy-C4-HSL to D1 cells (Miyamoto et al., 1996) is consistent with the

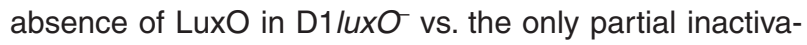
tion of LuxO by dephosphorylation on addition of 3hydroxy-C4-HSL. In addition, introduction of an excess of lux $R_{v h}$ to wild-type cells had little effect on luminescence, indicating that LuxO maintained a tight regulatory control on expression of lux $R_{v h}$ even when there were multiple copies of lux $R_{v h}$. In contrast, the cells produced more light (up to 10-fold) when lux $R_{v h}$ was added to the already strongly luminous luxO null mutants, consistent with loss of regulation of LuxO repression of $I u x R_{v h}$. These results indicate that the quorum-sensing signals are transmitted

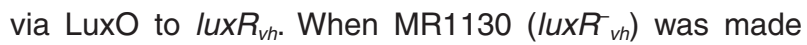
luxO', the dark phenotype remained unchanged: no other product necessary for luminescence appears to be repressed by LuxO. As LuxO is a $\sigma^{54}$ regulator (Lilley and Bassler, 2000), it is unlikely that it can bind directly to the $I_{u x} R_{v h}$ promoter. Whether or not a putative repressor $(\mathrm{X})$, synthesis of which may be controlled by LuxO, interacts directly with the lux $R_{v h}$ promoter is unknown; however, experiments are under way to detect proteins other than $\operatorname{LuxR}_{v h}$ that bind strongly to the $/ u x R_{v h}$ promoter. The possibility that LuxO may destabilize $\operatorname{lux}_{v h}$ mRNA cannot be ruled out, as both message and protein of $l u x R_{v h}$ would increase in a luxO mutant under these conditions. To determine whether another phenotype known to be under the control of $\mathrm{LuxR}_{\mathrm{vh}}$, namely PHB production late in growth, is associated with LuxO, MR1130 and D1 and their luxO null counterparts were analysed for the presence of crotonic acid, the oxidation product of $\mathrm{PHB}$.

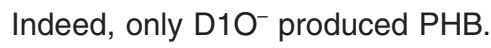

The possibility that LuxO control of lux $R_{v h}$ expression is a general regulatory mechanism was tested, because LuxO has been discovered to have similar effects on luminescence in V. fischeri (Miyamoto et al., 2000). Moreover, a LuxR $\mathrm{R}_{\mathrm{vh}}$ homologue, depicted as LitR, was recently isolated from V. fischeri ES114 (Fidopiastis et al., 2002). LitR is believed to upregulate LuxR, and the litR mutant PMF2 has one-fifth the luminescence expression of wild-type cells. Similar results were obtained for lit $R$ levels in $V$. fischeri wild type and the luxO- mutant compared with those with the same phenotypes from $V$. harveyi, with the litR mRNA being three times higher in luxO- than in wildtype $V$. fischeri (Table 1). When $V$. harveyi lux $R_{v h}$ was transferred into the lux $O^{-}$and wild-type $V$. fischeri cells, luminescence was stimulated only in the lux $0^{-}$cells in an analogous pattern to that found for the lux $0^{-}$mutant of $V$. harveyi. The luxO mutants of both $\mathrm{MJ}-211$ and $\mathrm{MJ}-215$ exhibited 10 - to 50 -fold higher luminescence as well as higher levels of litR mRNA than their isogenic parents, further substantiating the repressive role of LuxO in $V$. fischeri on luminescence and litR production.

Despite the fact that the two $V$. fischeri autoinducers, 3oxo-C6-HSL and C8-HSL, interact directly with $V$. fischeri luxR to activate lux operon transcription, we demonstrate here that the amount of litR mRNA was increased in the presence of additional C8-HSL in $V$. fischeri wild type, whereas the luxO counterpart remained elevated whether or not exogenous C8-HSL was present. In the autoinducer mutants, however, the effect of C8-HSL addition was less evident; however, their lux $0^{-}$counterparts had higher levels of litR mRNA, which were not altered in the presence of exogenous C8-HSL. These results are consistent with the autoinducer signal, at least in the case of C8-HSL, also being transduced through LuxO to inactivate LuxO repression of LitR in $V$. fischeri, in much the same way as 3-hydroxy-C4-HSL inactivates LuxO repression of LuxR $_{\mathrm{vh}}$ in $V$. harveyi. Consequently, a parallel system, as suggested by the homology between Ains and LuxM and the discovery of luxO in V. fischeri (Gilson et al., 1995; Miyamoto et al., 2000; Dunlap and Kita-Tsukamoto, 2001), appears to be present in both species.

It seems likely that other Vibrio species including nonluminescent species also use the LuxO-LuxR $\mathrm{R}_{\mathrm{vh}}$ regulatory system; recent evidence shows that $V$. cholerae LuxO represses HapR, the homologue of $L_{u x R}$, which in turn blocks tcpP gene expression and leads to inhibition of ToxR regulon expression. (Zhu et al., 2002). From independent searches (NCBI BLAST; Altschul et al., 1990) for $\mathrm{LuxR}_{\mathrm{vh}}$, LuxO and LuxU as well as a consensus region for LuxU and the C-terminus of LuxO, only the one Vibrio genome ( $V$. cholerae) in the database was found to contain LuxR $_{v h}$ and the LuxO-LuxU couple, which suggests that this system may be limited to bacteria in the genus Vibrio. Other Vibrio species, the genomes of which are not listed in the database, do have $\operatorname{LuxR}_{\mathrm{vh}}$ homologues, including OpaR of Vibrio parahaemolyticus (McCarter, 1998), SmcR of Vibrio vulnificus (McDougald et al., 2000; 2001; Shao and Hor, 2001), VanT of Vibrio anguillarum (Croxatto et al., 2002), as well as LitR of $V$. fischeri. With our demonstration of a link between $l u x R_{v h}$ and LuxO, it seems likely that these species will also be found to have LuxOU, which would raise the possibility that luxO is controlled by autoinducers produced by genes homologous to those of the $V$. harveyi system. 
Table 3. Bacteria used in this study.

\begin{tabular}{|c|c|c|c|}
\hline & Strain & Relevant features & Source or reference \\
\hline V. harveyi & B392 & $\operatorname{lu} x R_{v n}{ }^{+} \operatorname{lu} x O^{+}$ & Our laboratory \\
\hline V. harveyi & BB7 & $\operatorname{lux} R_{v i}{ }^{+} \operatorname{lu} x O^{+}$ & Gift from M. Silverman (Martin et al., 1989) \\
\hline V. harveyi & MR1130 & lux $R_{v h}{ }^{-}$of $\mathrm{BB} 7$ & Gift from M. Silverman (Martin et al., 1989) \\
\hline V. harveyi & D1 & Autoinducer mutant of B392 & Cao and Meighen (1989) \\
\hline V. harveyi & $\mathrm{VhO}^{-}$ & luxO of B392 & Miyamoto et al. (2000) \\
\hline V. harveyi & D10- & luxO of $\mathrm{D} 1$ & This study \\
\hline V. harveyi & $\mathrm{MRO}^{-}$ & luxO of MR1130 & This study \\
\hline V. fischeri & MJ-1 & 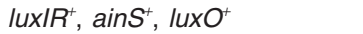 & Gift from B. L. Bassler \\
\hline V. fischeri & MJ-100 & luxIR $R^{+}$ainS $S^{+}, \operatorname{lit} R^{+}, \operatorname{lux} O^{+}$ & P. V. Dunlap (Kuo et al., 1994) \\
\hline V. fischeri & ES114 & 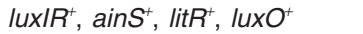 & E. Ruby (Fidopiastis et al. 2002) \\
\hline V. fischeri & $\mathrm{VfO}^{-}$ & $\operatorname{luxO}$ of $\mathrm{MJ}-1$ & Miyamoto et al. (2000) \\
\hline V. fischeri & MJ-211 & luxt of $\mathrm{MJ}-100$ & P. V. Dunlap (Kuo et al., 1994) \\
\hline V. fischeri & MJ-215 & luxt, ains of $\mathrm{MJ}-100$ & P.V. Dunlap (Kuo et al., 1994) \\
\hline E. coli & BL21(DE3) & Contains T7 RNA polymerase & Studier et al. (1990) \\
\hline
\end{tabular}

\section{Experimental procedures}

\section{Bacteria, plasmids and media}

Table 3 and Table 4 list the bacteria and plasmids, respectively, that were used in this study, their features and their sources or references. The marine bacteria studied were $V$. harveyi B392 (in our laboratory) and $V$. fischeri MJ-1 (a gift from B. L. Bassler). Escherichia coli BL-21(DE3) (Studier et al., 1990) transformed with either pT7 (Tabor and Richardson, 1985) or luxR $R_{v h}$ of $V$. harveyi B392 in pT7 (pT7/luxR $\left.\mathrm{R}_{\mathrm{vh}}\right)$ (Miyamoto et al., 1996) or crp in pT7 (pT7/crp) (Chatterjee et al., 2002) was grown in LB medium and treated with IPTG (Sigma) to induce the gene product placed after the pT7 promoter as described previously (Studier et al., 1990). The media used, LB or complex (Miyamoto et al., 2000), contained either $1 \%(\mathrm{w} / \mathrm{v}) \mathrm{NaCl}$ for $V$. harveyi $\mathrm{B} 392$ and its isogenic mutants as well as $E$. coli, or $2 \% \mathrm{NaCl}$ for $V$. harveyi BB7 and its isogenic mutants as well as $V$. fischeri. When indicated, MJ-1, MJ-211 (lux广; Kuo et al., 1994) and MJ-215 (lux广ainS; Kuo et al., 1996) were grown in ASH medium (Dunlap and Kuo, 1992). As the strains studied here have been determined to be resistant to ampicillin, the antibiotic was routinely added at $100 \mu \mathrm{g} \mathrm{ml}^{-1}$. The growth temperature was maintained at $28^{\circ} \mathrm{C}$ with good aeration. Spectrophotometry at $A_{660}$ was used to measure growth, and a custom-built luminometer measured luminescence where one light unit $(\mathrm{LU})=4 \times 10^{9}$ quanta $\mathrm{s}^{-1}$ (Hastings and Weber, 1963).

$\mathrm{D} 1$, an autoinducer mutant of $V$. harveyi B392, has been described previously (Cao and Meighen, 1989) as has MR1130, a lux $R_{v h}$ null mutant of $V$. harveyi BB7, a gift from M. Silverman (Martin et al., 1989). MJ-100 and MJ-211 are the isogenic wild-type and lux $\Gamma^{2}$ mutant, respectively, of $V$. fischeri (Kuo et al., 1994). As both MJ-100 and MJ-1 had the same phenotype, only data from $\mathrm{MJ}-1$ are reported here. Likewise, MJ-215 (lux广ainS ${ }^{-}$) was derived from MJ-211 (Kuo et al., 1996).

\section{Synthesis and purification of synthetic autoinducers}

N-3-hydroxybutanoyl-HSL (3-hydroxy-C4-HSL) was synthesized as described previously (Cao and Meighen, 1989). For $\mathrm{N}$-octanoyl-HSL (C8-HSL), $1 \mathrm{~g}$ of 1-ethyl-3-(3-dimethylaminopropyl)carbodimide (Sigma), $1 \mathrm{~g}$ of $\alpha$-amino- $\gamma$-butyrolactone hydrobromide (Aldrich), $0.6 \mathrm{ml}$ of octanoic acid (Aldrich, neat $0.91 \mathrm{~g} \mathrm{ml}^{-1}$ ) and $0.756 \mathrm{ml}$ of $5 \mathrm{M} \mathrm{NaOH}$ were mixed and adjusted to $10 \mathrm{ml}$ with $\mathrm{H}_{2} \mathrm{O}$. After overnight mixing at room

Table 4. Plasmids used in this study.

\begin{tabular}{|c|c|c|}
\hline Name & Features & Source or reference \\
\hline pMF-2 & litR of $V$. fischeri ES114, lux $R_{v h}$ homologue & Fidopiastis et al. (2002) \\
\hline pT7 & pT7 promoter plasmid & Tabor and Richardson (1985) \\
\hline pT7/luxR $\mathrm{vh}_{\mathrm{vh}}$ & lux $R_{v h}$ of $V$. harveyi B392 in pT7 & Miyamoto et al. (1996) \\
\hline pT7/crp & crp of V. harveyi B392 in pT7 & Chatterjee et al. (2002) \\
\hline pT7.litR & litR of $V$. fischeri ES114 in pT7 & Fidopiastis et al. (2002) \\
\hline pKT230 & $m o b^{+}$, broad-host-range vector, source of $k n^{R}$ & Bagdasarian et al. (1981) \\
\hline pMGM150 & luxR $R_{v h}$ of $V$. harveyi B392 in pKT230 & Miyamoto et al. (1996) \\
\hline pJQ200 & $m o b^{+}$, sacB, suicide vector & Quandt and Hynes (1993) \\
\hline pBluescript SK+ & cloning vector & Stratagene \\
\hline pBlue/luxC & promoter region of the $V$. harveyi lux operon in pBluescript & This study \\
\hline pVHO-2 & V. harveyi luxO with $k n^{R}$ interrupt in pJQ200 & Miyamoto et al. (2000) \\
\hline pVHO-4 & luxO PCR product of $V$. harveyi BB7 in pBluescript & This study \\
\hline pVHO-5 & pVHO-4 with $k n^{R}$ marker, luxO interrupt & This study \\
\hline pVHO-6 & VHO-5 in pJQ200 & This study \\
\hline pVFO-4 & V. fischeri luxO with $k n^{R}$ interrupt in pT7 & Miyamoto et al. (2000) \\
\hline pVFO-7 & VFO-4 in pJQ200 & This study \\
\hline
\end{tabular}


temperature, the sample was extracted three times using an equal volume of methylene chloride each time. After drying down, the sample was resuspended in $5 \%(\mathrm{v} / \mathrm{v})$ methanol in methylene chloride, followed by redrying. Methylene chloride $(10 \mathrm{ml})$ was added to the sample, which was applied to a $10 \mathrm{~cm} \times 2 \mathrm{~cm}$ diameter column of Kieselgel 60 (Merck), prewashed with methylene chloride. Flash chromatography was performed using stepwise additions of $1 \%, 2 \%$ and $5 \%$ $(\mathrm{v} / \mathrm{v})$ methanol in methylene chloride, and fractions of $10 \mathrm{ml}$ were collected. The C8-HSL was found in 1\% methanol after assays on thin-layer chromatography (TLC). The same fraction also gave rise to increased luminescence in MJ-211. After concentration, the synthetic C8-HSL was stored at $-20^{\circ} \mathrm{C}$ in methylene chloride at $600 \mu \mathrm{g} \mathrm{ml}^{-1}$.

\section{Gene replacement to create luxO null mutants}

All molecular microbiological techniques were performed according to Maniatis et al. (1982). The luxO mutants $V$. harveyi $\mathrm{B} 392$ and $V$. fischeri $\mathrm{MJ}-1$ were isolated previously (Miyamoto et al., 2000). The luxO- mutant of D1 was obtained with the use of pVHO-2 (Miyamoto et al., 2000). For the luxOmutant of MR1130 (MRO-), the polymerase chain reaction (PCR) product using two primers already described (Miyamoto et al., 2000) was first obtained using chromosomal DNA from $V$. harveyi BB7. The forward primer starts $272 \mathrm{bp}$ upstream of the ATG codon of luxO, and the reverse primer starts 10 codons from the end of the gene (Bassler et al., 1994b). The PCR product (after blunt-end digestion and then $\mathrm{BamHI}$ restriction) was ligated into pBluescript SK+ (Stratagene) at the EcoRV and BamHI sites (pVHO-4). The kanamycin resistance $\left(k n^{R}\right)$ marker was obtained from pKT230 after BamHI and DrallI and blunt-end digestions and ligated into $\mathrm{pVHO}-4$ at the unique $\mathrm{Ncol}$ site (blunt-ended), about $0.2 \mathrm{kbp}$ after the $5^{\prime}$-ATG start site of luxO (pVHO-5). The entire luxO and $k n^{R}$ interrupt was then excised with $X b a l$ and Sall of the polylinker of pVHO-5 and ligated into the corresponding sites, dephosphorylated, of the gentamicinresistant, sacB suicide vector pJQ200 (Quandt and Hynes, 1993) to create pVHO-6, which was then used to perform luxO gene replacement in MM1130. In order to create the luxO mutant of $\mathrm{MJ}^{-} 211$ and $\mathrm{MJ}^{-215}$, the plasmid pVFO-4, also described earlier, was restricted at the $\mathrm{Xbal}$ and $\mathrm{Sacl}$ sites to release the luxO fragment interrupted by the $k n^{R}$ marker, which was ligated to PJQ200, which had also been restricted at the same sites and treated with alkaline phosphatase. The desired plasmid pVFO-7 was mobilized into MJ211 to yield $\mathrm{MJ}-211 / \mathrm{uxO}$. Southern blot hybridization analyses of all luxO mutants confirmed successful gene replacement of the luxO allele with luxO DNA interrupted by the $\mathrm{kn}^{R}$ cassette and the loss of the DNA vector. For growth of the luxO null mutants, kanamycin $\left(40 \mu \mathrm{g} \mathrm{ml}^{-1}\right)$ and ampicillin $\left(100 \mu \mathrm{g} \mathrm{ml}^{-1}\right)$ were routinely added to media.

\section{Addition of lux $\mathrm{R}_{\mathrm{vh}}$ in trans to Vibrios}

Complementation of $\operatorname{lux}_{v h}$ in trans was carried out in $V$. harveyi and $V$. fischeri with pMGM150, which carries the luxR $_{v h}$ of $V$. harveyi $\mathrm{B} 392$ in the mobilization vector pKT230 (Bagdasarian et al., 1981) as described previously
(Miyamoto et al., 1996). As controls, pKT230 alone was transferred into the same strains. To maintain selective pressure, the LB medium included streptomycin at $40 \mu \mathrm{gll}^{-1}$.

\section{RNA dot-blot hybridization}

RNA was extracted from cellular pellets using the RNeasy kit (Qiagen) followed by DNase I digestion for $15 \mathrm{~min}$ at $37^{\circ} \mathrm{C}$ (Gibco BRL cat. no.18047-019). After phenol extraction and ethanol precipitation, RNA dot blots using the same amount of RNA for each sample were prepared (Miyamoto et al., 1996). DNA, ${ }^{32} \mathrm{P}$-labelled with random primers and $\left[\alpha-{ }^{32} \mathrm{P}\right]-$ dCTP (Amersham) in a Klenow reaction according to the manufacturer's specifications (NEB), was used as hybridization probe after heat denaturation. $V$. harveyi B392 lux $R_{v h}$ in pT7 $\left(\mathrm{pT} 7 / \mathrm{lux} \mathrm{R}_{\mathrm{vh}}\right)$ was the source for the lux $R_{v h}$ probe. The plasmid pMF-2 (litR ${ }^{+}$) was constructed by M. Fidopiastis of E. Ruby's laboratory (Fidopiastis et al., 2002). The EcoRI fragment containing litR was excised and inserted into pT7 (Tabor and Richardson, 1985), and the resultant plasmid (pT7/litR) was used as the probe for litR mRNA. For luxC DNA, the restricted DNA insert (Sacl and Clal) from cloned lux DNA in our laboratory (Swartzman and Meighen et al., 1993) was subcloned into pBluescript SK+ at the corresponding restriction sites (pBlue/luxC). The luxC DNA (0.45 kbp) encompasses the promoter and transcriptional start site of the lux operon and 62 bp of the luxC gene (Swartzman et al., 1992). Finally, the crp probe was obtained using pT7/crp (Chatterjee et al., 2002). The hybridized blots were visualized using the Fuji Bioimager. A control using E. coli RNA on each blot typically gave a background reading of $<70$ c.p.m., and the background was subtracted from the raw results of each set of blots. It should be noted that the specific activities of the probes varied with their base content and size, and comparison of intensity levels of mRNA among them cannot be done.

\section{Mobility shift analysis}

The plasmid $\mathrm{pT} 7 / \mathrm{luxR}$ vh was restricted with Sacl and Mfel to provide a 400 bp DNA fragment that contains the promoter region of $l u x R_{v h}$ (Chatterjee et al., 1996). After a Klenow fillin reaction (NEB) with $\left[\alpha^{-32} \mathrm{P}\right]$-dATP (Amersham) at the Mfel site, the DNA was subjected to mobility shift analysis after mixing with various cell-free crude lysates prepared from cellular pellets of cells grown in LB to a density of $A_{660}=0.6$, as described previously (Swartzman and Meighen, 1993). Protein determinations were performed using the Bio-Rad protein assay kit.

\section{Detection of polyhydroxybutyrate (PHB)}

A constant amount of cells (10 $\mathrm{A}_{660}$ units) grown in complex medium was harvested at high cell densities $\left(A_{660}=3\right)$ and processed for $\mathrm{PHB}$ using $\mathrm{NaOCl}$ oxidation as described previously (Sun et al., 1994; Miyamoto et al., 1998). Wavelength scans were obtained using the Beckman DU-640 spectrophotometer. 


\section{Acknowledgements}

This work was supported by a grant, MT-7672, from the Canadian Institute of Health Research (CIHR). P. V. Dunlap acknowledges support from DARPA grant F30602-01-20563. We wish to thank Dr Pat M. Fidopiastis for his expertise and affable communication.

\section{References}

Altschul, S.F., Gish, W., Miller, W., Myers, E.W., and Lipman, D.J. (1990) Basic local alignment search tool. J Mol Biol 215: 403-410.

Bagdasarian, M., Lurz, R., Rückert, B., Franklin, F.C.H., Bagdasarian, M.M., Frey, J., and Timmis, K.N. (1981) Specific-purpose plasmid cloning vectors. II. Broad host range, high copy number, RSF1010-derived vectors, and a hostvector system for gene cloning in Pseudomonas. Gene 16: 237-247.

Bassler, B.L. (1999) How bacteria talk to each other: regulation of gene expression by quorum sensing. Curr Opin Microbiol 2: 582-587.

Bassler, B.L., Wright, M., Showalter, R.E., and Silverman, M.R. (1993) Intercellular signalling in Vibrio harveyi: sequence and function of genes regulating expression of luminescence. Mol Microbiol 9: 773-786.

Bassler, B.L., Wright, M., and Silverman, M.R. (1994a) Multiple signalling systems controlling expression of luminescence in Vibrio harveyi: sequence and function of genes encoding a second sensory pathway. Mol Microbiol 13: 273-286.

Bassler, R.L., Wright, M., and Silverman, M.R. (1994b) Sequence and function of LuxO, a negative regulator of luminescence in Vibrio harveyi. Mol Microbiol 12: 403-412.

Bassler, B.L., Greenberg, E.P., and Stevens, A.M. (1997) Cross-species induction of luminescence in the quorumsensing bacterium Vibrio harveyi. J Bacteriol 179: 40434045.

Cao, J.-G., and Meighen, E.A. (1989) Purification and structural identification of an autoinducer for the luminescence system of Vibrio harveyi. J Biol Chem 264: 21670-21676.

Cao, J.-G., and Meighen, E.A. (1993) Biosynthesis and stereochemistry of the autoinducer controlling luminescence in Vibrio harveyi. J Bacteriol 175: 3856-3862.

Chatterjee, J., Miyamoto, C.M., and Meighen, E.A. (1996) Autoregulation of luxR: the Vibrio harveyi lux-operon activator functions as a repressor. Mol Microbiol 20: 415-425.

Chatterjee, J., Miyamoto, C.M., Zouzoulas, A., Lang, B.F., Skouris, N., and Meighen, E.A. (2002) MetR and CRP bind to the Vibrio harveyi lux promoters and regulate luminescence. Mol Microbiol 46: 101-111.

Chen, X., Schauder, S., Potier, N., Van Dorsselaer, A., Pelczer, I., Bassler, B.L., and Hughson, F.M. (2002) Structural identification of a bacterial quorum-sensing signal containing boron. Nature 415: 545-549.

Croxatto, A., Chalker, V.J., Lauritz, J., Jass, J., Hardman, A., Williams, P., et al. (2002) VanT, a homologue of Vibrio harveyi LuxR, regulates serine, metalloprotease, pigment, and biofilm production in Vibrio anguillarum. $J$ Bacteriol 184: 1617-1629.

Dunlap, P.V., and Kita-Tsukamoto (2001) Luminous bacteria.
In The Prokaryotes, an Evolving Electronic Resource for the Microbiological Community. Dworkin, M., Falkow, S., Rosenberg, E., Schleifer, K.-H., Stackebrandt, E. (eds). New York: Springer-Verlag.

Dunlap, P.V., and Kuo, A. (1992) Cell density-dependent modulation of the Vibrio fischeri luminescence system in the absence of autoinducer and LuxR protein. J Bacteriol 174: 2440-2448.

Engebrecht, J., and Silverman, M. (1984) Identification of genes and gene products necessary for bacterial bioluminescence. Proc Natl Acad Sci USA 81: 4154-4158.

Engebrecht, J., Nealson, K., and Silverman, M. (1983) Bacterial bioluminescence: isolation and genetic analysis of functions from Vibrio fischeri. Cell 32: 773-781.

Fidopiastis, P.M., Miyamoto, C.M., Jobling, M.G., Meighen, E.A., and Ruby, E.G. (2002) LitR, a new transcriptional activator in Vibrio fischeri, regulates luminescence and symbiotic light organ colonization. Mol Microbiol 45: 131143.

Freeman, J.A., and Bassler, B.L. (1999a) A genetic analysis of the function of LuxO, a two-component response regulator involved in quorum sensing in Vibrio harveyi. Mol Microbiol 31: 665-677.

Freeman, J.A., and Bassler, B.L. (1999b) Sequence and function of LuxU: a two-component phosphorelay protein that regulates quorum sensing in Vibrio harveyi. J Bacteriol 181: 899-906.

Freeman, J.A., Lilley, B.N., and Bassler, B.L. (2000) A genetic analysis of the functions of LuxN: a two-component hybrid sensor kinase that regulates quorum sensing in Vibrio harveyi. Mol Microbiol 35: 139-149.

Fuqua, W.C., Winans, S.C., and Greenberg, E.P. (1994) Quorum sensing in bacteria: the LuxR-Luxl family of cell density-responsive transcriptional regulators. J Bacteriol 176: 269-275.

Gilson, L., Kuo, A., and Dunlap, P.V. (1995) AinS and a new family of autoinducer synthesis proteins. J Bacteriol 177: 6946-6951.

Hanzelka, B.L., Parsek, M.R., Val, D.L., Dunlap, P.V., Cronan, J.E., Jr, and Greenberg, E.P. (1999) Acylhomoserine lactone synthase activity of the Vibrio fischeri AinS protein. J Bacteriol 181: 5766-5770.

Hastings, J.W., and Weber, G. (1963) Total quantum flux of isotopic sources. J Opt Soc Am 53: 1410-1415.

Jobling, M.G., and Holmes, R.K. (1997) Characterization of hapR, a positive regulator of the Vibrio cholerae $\mathrm{HA} /$ protease gene hap, and its identification as a functional homologue of the Vibrio harveyi luxR gene. Mol Microbiol 26: 1023-1034.

Joyce, E.A., Bassler, B.L., and Wright, A. (2000) Evidence for a signaling system in Helicobacter pylori: detection of a luxS-encoded autoinducer. J Bacteriol 182: 3638-3643.

Kuo, A., Blough, N.V., and Dunlap, P.V. (1994) Multiple Nacyl-L-homoserine lactone autoinducers of luminescence in the marine symbiotic bacterium Vibrio fischeri. $J$ Bacteriol 176: 7558-7565.

Kuo, A., Callahan, S.M., and Dunlap, P.V. (1996) Modulation of luminescence operon expression by N-octanoyl-Lhomoserine lactone in ains mutants of Vibrio fischeri. $J$ Bacteriol 178: 971-976.

Lilley, B.N., and Bassler, B.L. (2000) Regulation of quorum 
sensing in Vibrio harveyi by LuxO and sigma-54. Mol Microbiol 36: 940-954.

McCarter, L.L. (1998) OpaR, a homolog of Vibrio harveyi LuxR, controls opacity of Vibrio parahaemolyticus. J Bacteriol 180: 3166-3173.

McDougald, D., Rice, S.A., and Kjelleberg, S. (2000) The marine pathogen Vibrio vulnificus encodes a putative homologue of the Vibrio harveyi regulatory gene, luxR: a genetic and phylogenetic comparison. Gene 248: 213221.

McDougald, D., Rice, S.A., and Kjelleberg, S. (2001) SmcRdependent regulation of adaptive phenotypes in Vibrio vulnificus. J Bacteriol 183: 758-762.

Maniatis, T., Fritsch, E.F., and Sambrook, J. (1982) Molecular Cloning: a Laboratory Manual. Cold Spring Harbor, NY: Cold Spring Harbor Laboratory Press.

Martin, M., Showalter, R., and Silverman, M. (1989) Identification of a locus controlling expression of luminescence genes in Vibrio harveyi. J Bacteriol 171: 2406-2414.

Miyamoto, C.M., Smith, E.E., Swartzman, E., Cao, J.-G., Graham, A.F., and Meighen, E.A. (1994) Proximal and distal sites bind LuxR independently and activate expression of the Vibrio harveyi lux operon. Mol Microbiol 14: 255-262.

Miyamoto, C.M., Chatterjee, J., Swartzman, E., Szittner, R., and Meighen, E.A. (1996) The role of the lux autoinducer in regulating luminescence in Vibrio harveyi; control of luxR expression. Mol Microbiol 19: 767-775.

Miyamoto, C.M., Sun, W., and Meighen, E.A. (1998) The LuxR regulator protein controls synthesis of polyhydroxybutyrate in Vibrio harveyi. Biochim Biophys Acta 1384: 356-364.

Miyamoto, C.M., Lin, Y.H., and Meighen, E.A. (2000) Control of bioluminescence in Vibrio fischeri by the LuxO signal response regulator. Mol Microbiol 36: 594-607.

Quandt, J., and Hynes, M.F. (1993) Versatile suicide vectors which allow direct selection for gene replacement in gramnegative bacteria. Gene 127: 15-21.

Schauder, S., Shokat, K., Surette, M.G., and Bassler, B.L.
(2001) The LuxS family of bacterial autoinducers: biosynthesis of a novel quorum-sensing signal molecule. Mol Microbiol 41: 463-476.

Shao, C.-P., and Hor, L.-I. (2001) Regulation of metalloprotease gene expression in Vibrio vulnificus by a Vibrio harveyi LuxR homologue. J Bacteriol 183: 1369-1375.

Showalter, R.E., Martin, M.O., and Silverman, M.R. (1990) Cloning and nucleotide sequence of $l u x R$, a regulatory gene controlling bioluminescence in Vibrio harveyi. J Bacteriol 172: 2946-2954.

Studier, F.W., Rosenberg, A.H., Dunn, J.J., and Dubendorff, J.W. (1990) Use of T7 RNA polymerase to direct expression of cloned genes. Methods Enzymol 185: 60-89.

Sun, W., Cao, J.-G., Teng, K., and Meighen, E.A. (1994) Biosynthesis of poly-3-hydroxybutyrate in the luminescent bacterium, Vibrio harveyi, and regulation by the lux autoinducer, N-(3-hydroxybutanoyl) homoserine lactone. J Biol Chem 269: 20785-20790.

Swartzman, E., and Meighen, E.A. (1993) Purification and characterization of a poly (dA-dT) lux-specific DNA-binding protein from Vibrio harveyi and identification as LuxR. $J$ Biol Chem 268: 16706-16716.

Swartzman, E., Silverman, M., and Meighen, E.A. (1992) The luxR gene product of Vibrio harveyi is a transcriptional activator of the lux promoter. J Bacteriol 174: 74907493.

Swift, S., Williams, P., and Stewart, G.S.A.B. (1999) N-acylhomoserine lactones and quorum sensing in proteobacteria. In Cell-Cell Signaling in Bacteria. Dunny, G.M., and Winans, S.C. (eds). Washington, DC: American Society for Microbiology Press, 291-313.

Tabor, S., and Richardson, C.C. (1985) A bacteriophage T7 RNA polymerase/promoter system for controlled exclusive expression of specific genes. Proc Natl Acad Sci USA 82: 1074-1078.

Zhu, J., Miller, M.B., Vance, R.E., Dziejman, M., Bassler, B.L., and Mekalanos, J.J. (2002) Quorum-sensing regulators control virulence gene expression in Vibrio cholerae. Proc Natl Acad Sci USA 99: 3129-3134. 